\title{
Comparison of sevoflurane and propofol anesthesia on the incidence of hyperglycemia in patients with type 2 diabetes undergoing lung surgery
}

\author{
Hyuckgoo Kim, Jisoo Han, Sung Mee Jung, Sang-Jin Park, Nyeong Keon Kwon \\ Department of Anesthesiology and Pain Medicine, Yeungnam University College of Medicine, Daegu, Korea
}

\begin{abstract}
Background: The type and regimen of anesthesia may affect perioperative hyperglycemia following major surgical stress. This study compared the effects of sevoflurane and propofol on the incidence of hyperglycemia and clinical outcomes in diabetic patients undergoing lung surgery.

Methods: This retrospective study included 176 patients with type 2 diabetes mellitus who had undergone lung surgery. Blood glucose levels and clinical outcomes from the preoperative period to the first 2 postoperative days (PODs) were retrospectively examined in patients who received sevoflurane (group $S, n=87$ ) and propofol (group $P, n=89$ ) for maintenance of general anesthesia. The primary endpoint was the incidence of persistent hyperglycemia ( 2 consecutive blood glucose levels $>180 \mathrm{mg} / \mathrm{dL}$ [ $10.0 \mathrm{mmol} / \mathrm{L}]$ ) during the perioperative period. The secondary composite endpoint was the incidence of major postoperative complications and 30-day mortality rate after surgery.

Results: Blood glucose levels similarly increased from the preoperative period to the second POD in both groups $(p=0.857)$. Although blood glucose levels at 2 hours after surgery were significantly lower in group $P$ than in group $S(p=0.022 ; 95 \%$ confidence interval for mean difference, -27.154 to -2.090$)$, there was no difference in the incidence of persistent hyperglycemia during the perioperative period (group S, 70\%; group $\mathrm{P}, 69 \% ; p=0.816$ ). The composite of major postoperative complications and all-cause in-hospital and 30-day mortality rates were also comparable between the two groups.
\end{abstract}

Conclusion: Sevoflurane and propofol were associated with a comparable incidence of perioperative hyperglycemia and clinical outcomes in diabetic patients undergoing lung surgery.

Keywords: Diabetes mellitus; General anesthesia; Lung surgery; Propofol; Sevoflurane

\section{INTRODUCTION}

Glucose homeostasis in surgical patients with diabetes mellitus (DM) can be affected by the complex interaction of several factors in the perioperative period. Nutritional deprivation and disruption of usual anti-DM management frequently

Received: March 26, 2018, Revised: May 10, 2018 Accepted: May 15, 2018

Corresponding Author: Sung Mee Jung, Department of Anesthesiology and Pain Medicine, Yeungnam University College of Medicine, 170, Hyeonchung-ro, Nam-gu, Daegu 42415, Korea

Tel: +82-53-620-3354, Fax: +82-53-626-5275

E-mail: applejsm@gmail.com complicates glycemic control. Surgical stress results in the excessive release of proinflammatory cytokines and a sympathetic activation-related rise in catecholamine, cortisol, glucagon, and growth hormone levels. This elevation in counter-regulatory hormones leads to the elevation of blood glucose levels by facilitating endogenous glucose production and insulin resistance in the liver, and reducing glucose utilization in the skeletal muscle [1]. The development of insulin resistance and impaired insulin signaling during surgery are more pronounced during the early postoperative period, and may persist for days or weeks following surgery [2].

Acute hyperglycemia is associated with an increased risk for surgical morbidity and mortality in patients undergoing

Copyright (C) 2018 Yeungnam University College of Medicine

This is an Open Access article distributed under the terms of the Creative Commons Attribution Non-Commercial License (http://creativecommons.org/licenses/by-nc/4.0/) which permits unrestricted non-commercial use, distribution, and reproduction in any medium, provided the original work is properly cited. 
major surgery independent of DM [3]. In particular, persistent hyperglycemia during the perioperative period is associated with a higher postoperative adverse event rate than transient hyperglycemia in patients with DM [3,4]. Because perioperative hyperglycemia is a potentially reversible cause of poor surgical outcome, anesthesiologists should optimize the management of blood glucose levels to improve perioperative care in these patients.

Generally, the degree of perioperative hyperglycemia is dependent on the preoperative metabolic state of the patient, the stress response evoked by surgical injury, insulin resistance, as well as intraoperative management including medications and fluid [5]. Anesthesia also modulates glycemic response by affecting neuroendocrine surgical response or directly modifying pancreatic insulin release, even in absence of surgical stress [1,6]. Several studies have indicated that volatile anesthetics exacerbate hyperglycemia by suppressing glucosestimulated insulin secretion, and impairing glucose utilization and clearance [7-10]. On the other hand, propofol has been reported to promote insulin resistance, but concomitantly increases insulin secretion and preserves glucose utilization in animal models [9-12]. A few clinical studies have reported that propofol anesthesia resulted in lower blood glucose levels compared with volatile anesthesia during surgery in non-DM patients $[13,14]$. However, no studies have examined the effect of volatile anesthetic versus propofol on blood glucose levels throughout the perioperative period, and postoperative outcomes in DM patients undergoing major surgery.

Accordingly, in this retrospective study, we compared the effects of sevoflurane and propofol on the incidence of persistent hyperglycemia during the perioperative period, and on postoperative morbidity and mortality in DM patients undergoing open lung surgery.

\section{MATERIALS AND METHODS}

This study was approved by the Yeungnam University Hospital Institutional Review Board (YUMC 2015-08-003). Given the retrospective nature of the study and the use of anonymized patient data, requirements for informed written consent were waived. A retrospective chart analysis of adult patients with type $2 \mathrm{DM}$ who underwent general anesthesia for elective open lung surgery using one-lung ventilation (OLV) at a single center between January 2008 and May 2015 was conducted. A review of hospital medical and anesthetic records was performed to delete erroneously entered data and remove patient data with incomplete information. All patients with type $2 \mathrm{DM}$, who underwent surgery between 08:30 and 09:00 am, were included. Patients with concomitant acute or chronic renal failure requiring dialysis, emergent surgery, combined thoracic and abdominal surgery, thoracoscopic surgery, or requiring postoperative mechanical ventilation were excluded. Patient data were categorized into two groups based on the anesthetic agent used for maintenance of general anesthesia during lung surgery: the sevoflurane group (group S); and the propofol group (group P).

According to institutional protocol, the aim of glucose control management in DM patients undergoing surgery is to maintain glucose levels of 120 to $180 \mathrm{mg} / \mathrm{dL}$ (6.7 to $10.0 \mathrm{mmol} / \mathrm{L}$ ) in the perioperative period. Oral hypoglycemic agent (OHA) medication was continued up until the night before surgery and withheld on the morning of surgery. Long-acting insulin treatment was replaced by intravenous (IV) short-acting insulin and dextrose-containing fluid on the morning of surgery.

Patients were premedicated with an intramuscular injection of glycopyrrolate $0.2 \mathrm{mg} 30$ minutes before surgery. Anesthesia was induced by IV injection of propofol $1-1.5 \mathrm{mg} / \mathrm{kg}$ and continuous infusion of remifentanil at a rate of 0.05-0.1 $\mu \mathrm{g} / \mathrm{kg} / \mathrm{min}$. A double-lumen tube was used to facilitate oro-tracheal intubation after administration of IV rocuronium 0.75 $\mathrm{mg} / \mathrm{kg}$. Anesthesia was maintained by administration of sevoflurane or propofol, both combined with a continuous infusion of remifentanil at a rate of $0.05-0.1 \mu \mathrm{g} / \mathrm{kg} / \mathrm{min}$. The choice of anesthetic was at the full discretion of the attending anesthesiologists. Anesthetic depth during surgery was maintained in the range of 40-60 according to bispectral index monitoring during surgery. No glucose-containing fluid was administered during anesthesia. Patient-controlled IV analgesia with fentanyl or local infiltration with $0.5 \%$ ropivacaine for postoperative pain management was also selected at the discretion of the attending anesthesiologists. The IV opioids were injected when visual analogue pain scale scores exceeded 5. Patients were permitted meals on the morning of postoperative day (POD) 1.

Blood glucose levels were monitored with the following frequency, according to institutional protocol for DM patients undergoing surgery: on the morning before surgery; every 1 or 2 hours during surgery; at 2 hours after surgery; 
and every 6 hours thereafter until 2 days after surgery. Perioperative hyperglycemia was defined as 2 consecutive blood glucose levels $>180 \mathrm{mg} / \mathrm{dL}(10.0 \mathrm{mmol} / \mathrm{L})$ during the perioperative period and managed with regular IV insulin. Patients who took OHA medication before surgery were managed with OHA as usual, or IV regular insulin in case of uncontrolled hyperglycemia the day after surgery. Hypoglycemia was defined as blood glucose levels $<80 \mathrm{mg} / \mathrm{dL}$ ( $4.4 \mathrm{mmol} / \mathrm{L}$ ), which are known to be potentially detrimental to brain metabolism [15]. If hypoglycemia developed, treatment consisted of IV administration of $20 \%$ dextrose or oral intake of fluids containing glucose. The multiple blood glucose levels measured during PODs 1 and 2 were averaged for each day.

Demographic information, medical history, anesthetic and surgical data, laboratory tests, and postoperative outcomes of the patients were obtained from the medical records. The primary endpoint was the incidence of persistent hyperglycemia from the intraoperative period to POD 2. The secondary endpoints included the composite of major postoperative complications, such as surgical site infection (SSI), myocardial infarction (MI), cerebral infarction, acute respiratory distress syndrome (ARDS), and acute renal failure requiring hemodialysis during admission, and in-hospital and 30-day mortality rates.
Sample size was calculated using $G^{*}$ Power version 3.1.5 (free download, www.ResearchGate.net). It was ascertained that 87 patients were required in each group to detect a $25 \%$ intergroup difference in the incidence of persistent hyperglycemia during the perioperative period with an $\alpha$ of 0.05 and a power of $90 \%$. Statistical analysis was performed using IBM SPSS version 22.0 (IBM Co., Armonk, NY, USA). Categorical data are expressed as the number of patients (\%) and analyzed using the chi-squared test or Fischer's exact test, as appropriate. Continuous data are expressed as mean \pm standard deviation (SD) for normally distributed variables and median with interquartile range for non-normally distributed variables, and analyzed using the independent Student's t-test and the Mann-Whitney U test, respectively. The changes in blood glucose levels during the perioperative period between the two groups were compared using repeated measures ANOVA; A $p<0.05$ was considered to be statistically significant.

\section{RESULTS}

Of 188 adult patients with type $2 \mathrm{DM}$ undergoing open lung surgery under general anesthesia between January 2008 and May 2015 at a single center, 12 were excluded due to

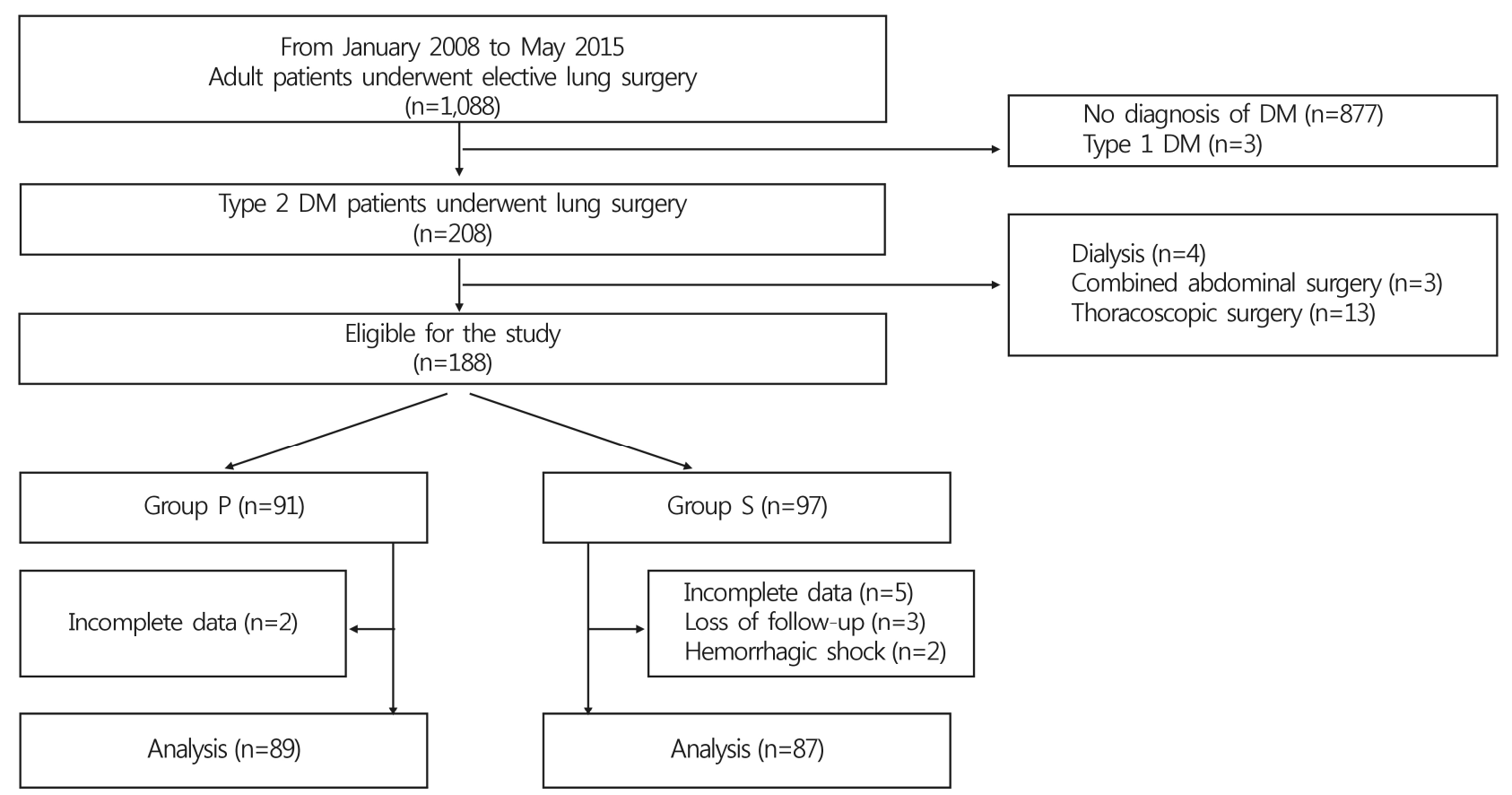

Fig. 1. Flow chart of the study design. DM, diabetes mellitus; Group P, propofol; Group S, sevoflurane. 
complete loss of preoperative or intraoperative glucose data $(\mathrm{n}=7)$, loss to follow-up $(\mathrm{n}=3)$, or hemorrhagic shock $(\mathrm{n}=2)$ (Fig. 1). Consequently, data from 87 patients who received sevoflurane inhalation and 89 who received propofol infusion for maintenance of general anesthesia during surgery were included in the final analysis. Preoperative patient characteristics, medical history, anesthetic and surgical data, and postoperative pain control regimens were comparable between the two groups (Table 1, 2). In particular, perioperative vital signs, including blood pressure, heart rate and peripheral oxygen saturation, and postoperative pain scales were comparable between the two groups. Thirteen (14.9\%) and 15 (16.9\%) patients in group $\mathrm{S}$ and $\mathrm{P}$, respectively, received long-acting insulin to control blood glucose levels preoperatively. Mean preoperative blood glucose levels between the two groups were comparable (group S, $132.4 \pm 40.9 \mathrm{mg} / \mathrm{dL}$; group P, $142.7 \pm$ $36.8 \mathrm{mg} / \mathrm{dL} ; p=0.074$ ) (Table 3). Although a significant intergroup difference in mean glucose level was observed 2 hours postoperatively (group S, $212.1 \pm 44.0 \mathrm{mg} / \mathrm{dL}$; group P, $197.5 \pm$ $40.0 \mathrm{mg} / \mathrm{dL} ; p=0.022 ; 95 \%$ confidence interval for mean differences, -27.154 to -2.090 ), changes in mean blood glucose levels were not different between the two groups over time in the perioperative period $(p=0.857)$ (Table 3 ).

There were no differences in the incidence of hyperglycemia at each time interval between the two groups (Fig. 2). Persistent hyperglycemia throughout the perioperative period was observed in 62 patients (70\%) in group $S$ and 62 patients (69\%) in group $\mathrm{P}(p=0.816)$. IV insulin use for glycemic control from surgery to POD 2 was also comparable between the two groups (Table 2). Six patients (3.4\%) during surgery and five patients (2.8\%) after surgery exhibited hypoglycemia (blood glucose level $<80 \mathrm{mg} / \mathrm{dL}$ [4.4 mmol/L]), without an intergroup difference. However, no patients with hypoglycemia received IV glucose because all blood glucose levels were $>70$ $\mathrm{mg} / \mathrm{dL}(3.9 \mathrm{mmol} / \mathrm{L})$ and increased $>80 \mathrm{mg} / \mathrm{dL}(4.4 \mathrm{mmol} / \mathrm{L})$ on repeated glucose check.

Table 1. Preoperative characteristics of patient

\begin{tabular}{|c|c|c|c|}
\hline & Group $\mathrm{P}^{\mathrm{a})}(\mathrm{n}=89)$ & Group $S^{\mathrm{b})}(\mathrm{n}=87)$ & $p$-value \\
\hline Age (y) & $64.8 \pm 9.9$ & $62.1 \pm 8.5$ & 0.053 \\
\hline Gender (M/F) & $67 / 22$ & $69 / 18$ & 0.524 \\
\hline $\mathrm{BMI}\left(\mathrm{kg} / \mathrm{m}^{2}\right)$ & $23.4 \pm 3.1$ & $22.9 \pm 3.0$ & 0.274 \\
\hline ASA PS (I/II/III) & $0 / 59 / 30$ & $2 / 62 / 23$ & 0.252 \\
\hline \multicolumn{4}{|l|}{ Comorbid status (n) } \\
\hline Hypertension & $55(61.8)$ & $46(52.9)$ & 0.231 \\
\hline CHF within 30 days & 0 & $2(2.3)$ & 0.243 \\
\hline Atrial fibrillation & $3(3.4)$ & 0 & 0.246 \\
\hline Renal insufficiency ${ }^{c}$ & $9(10.1)$ & $3(3.4)$ & 0.079 \\
\hline \multicolumn{4}{|l|}{ Preoperative medication } \\
\hline$\beta$-blocker & $9(10.1)$ & $9(10.8)$ & 0.959 \\
\hline Calcium Channel Blocker & $27(30.3)$ & $25(28.7)$ & 0.816 \\
\hline $\mathrm{ARB} / \mathrm{ACEI}$ & $29(32.6)$ & $21(24.1)$ & 0.214 \\
\hline Aminophylline & $6(6.7)$ & $1(1.1)$ & 0.118 \\
\hline Steroid & $1(1.1)$ & $1(1.1)$ & 0.987 \\
\hline Fasting blood glucose (mg/dL) & $144.5 \pm 33.2$ & $135.8 \pm 42.3$ & 0.131 \\
\hline Preoperative glycemic control (n) & & & 0.510 \\
\hline No medication & $8(9.0)$ & $5(5.7)$ & \\
\hline Insulin & $12(13.5)$ & $7(8.0)$ & \\
\hline $\mathrm{OHA}$ & $60(67.4)$ & $64(73.6)$ & \\
\hline Insulin+OHA & $9(10.1)$ & $11(12.6)$ & \\
\hline
\end{tabular}

Data are presented as the mean \pm standard deviation or number (\%).

BMI, body mass index; ASA PS, American Society of Anesthesiologists physical status; CHF, congestive heart failure; ARB, angiotensin receptor blocker; ACEI, angiotensin-converting enzyme inhibitor; OHA, oral hypoglycemic agent.

${ }^{a}$ Group P, propofol. ${ }^{\text {b) }}$ Group S, sevoflurane. ${ }^{\mathrm{c}}$ Renal insufficiency is defined as serum creatinine $>2 \mathrm{mg} / \mathrm{dL}$. 
Table 2. Anesthetic and surgical data

\begin{tabular}{|c|c|c|c|}
\hline & Group $\mathrm{P}^{\mathrm{a})}(\mathrm{n}=89)$ & Group $S^{b)}(n=87)$ & $p$-value \\
\hline Type of surgery (n) & & & 0.363 \\
\hline Lobectomy & $56(66.3)$ & $48(55.2)$ & \\
\hline Pneumonectomy & $4(4.5)$ & $4(4.6)$ & \\
\hline Lung decortication & $3(3.4)$ & $7(8.0)$ & \\
\hline Wedge resection & $23(25.8)$ & $28(32.2)$ & \\
\hline Remifentanil ( $\mu \mathrm{g} / \mathrm{kg} / \mathrm{min})$ & $0.09 \pm 0.02$ & $0.10 \pm 0.03$ & 0.148 \\
\hline Crystalloid intake (mL) & $1,830 \pm 1,064$ & $1,952 \pm 1,308$ & 0.499 \\
\hline Colloid intake $(\mathrm{mL})$ & $114 \pm 222$ & $90 \pm 186$ & 0.443 \\
\hline Urine output $(\mathrm{mL})$ & $421 \pm 317$ & $570 \pm 483$ & 0.024 \\
\hline Transfusion (n) & $13(14.6)$ & $7(8.0)$ & 0.170 \\
\hline Operation duration (min) & $203 \pm 105$ & $196 \pm 104$ & 0.684 \\
\hline Anesthesia duration (min) & $279 \pm 114$ & $265 \pm 107$ & 0.412 \\
\hline OLV duration (min) & $164 \pm 85$ & $151 \pm 87$ & 0.359 \\
\hline Postoperative pain control (n) & & & 0.296 \\
\hline IV PCA & $53(59.6)$ & $45(51.7)$ & \\
\hline Wound infiltration & $36(40.4)$ & $42(48.3)$ & \\
\hline \multicolumn{4}{|l|}{ Insulin use (n) } \\
\hline During surgery & $13(14.6)$ & $5(8.4)$ & 0.052 \\
\hline Operative day after surgery & $20(23.3)$ & $27(31.0)$ & 0.250 \\
\hline POD 1 & $43(48.3)$ & $45(51.7)$ & 0.651 \\
\hline POD 2 & $36(40.4)$ & $41(48.2)$ & 0.301 \\
\hline \multicolumn{4}{|l|}{ Hypoglycemia (n) } \\
\hline During surgery & $1(1.1)$ & $5(5.7)$ & 0.116 \\
\hline After surgery-2nd POD & $2(2.2)$ & $3(3.4)$ & 0.680 \\
\hline
\end{tabular}

Data are presented as the mean \pm standard deviation or number (\%).

OLV, one-lung ventilation; IV PCA, intravenous patient-controlled analgesia; POD, postoperative day.

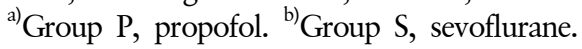

Postoperative outcomes are summarized in Table 4. There were no differences in the occurrence of SSI, cerebral infarction, MI, ARDS, or renal failure requiring hemodialysis during hospitalization between the two groups. All-cause in-hospital and 30-day mortality rates were also comparable between the two groups.

\section{DISCUSSION}

This retrospective study demonstrated that anesthesia using sevoflurane or propofol resulted in a comparable incidence of persistent hyperglycemia during the perioperative period in patients with type $2 \mathrm{DM}$ undergoing major thoracic surgery. Although the mean blood glucose level in the immediate postoperative period was lower after propofol anesthesia, it did not result in improved postoperative outcome or

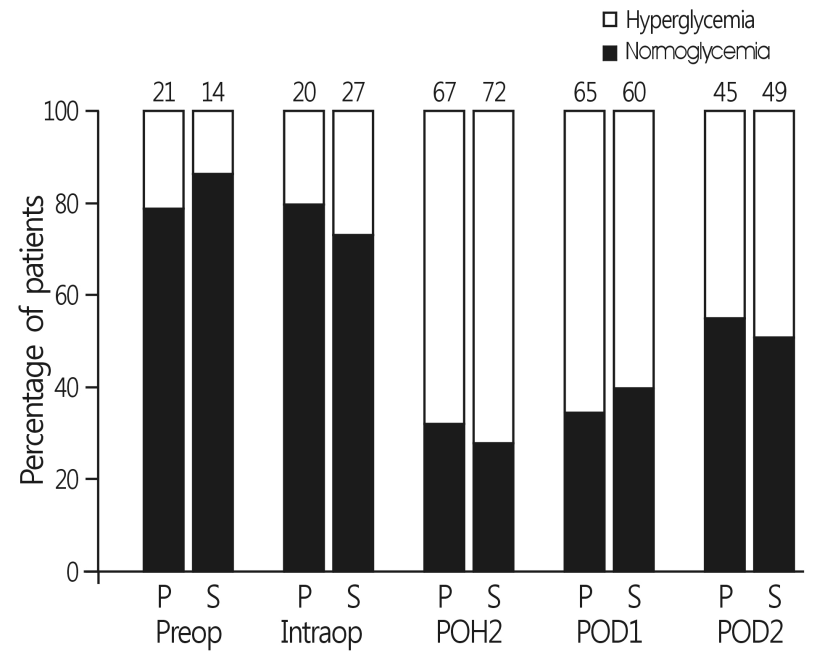

Fig. 2. Incidence of hyperglycemia during the perioperative period. Numbers are expressed as percentages of hyperglycemic patients. Group P, propofol; Group S, sevoflurane; Preop, before surgery; Intraop, during surgery; $\mathrm{POH}$, postoperative hour; $\mathrm{POD}$, postoperative day. 
Table 3. Blood glucose levels during perioperative period

\begin{tabular}{lccc}
\hline & $\begin{array}{c}\text { Group } P^{\mathrm{a})} \\
(\mathrm{n}=89)\end{array}$ & $\begin{array}{c}\left.\text { Group } S^{\mathrm{b}}\right) \\
(\mathrm{n}=87)\end{array}$ & $p$-value \\
\hline $\begin{array}{l}\text { Operation day } \\
\text { Before surgery }\end{array}$ & $142.7 \pm 36.8$ & $132.4 \pm 40.9$ & 0.074 \\
During surgery & & & \\
$\quad$ 1 hour & $157.9 \pm 36.1$ & $143.4 \pm 50.8$ & 0.212 \\
$\quad$ 2-3 hours & $145.5 \pm 35.9$ & $149.6 \pm 40.8$ & 0.550 \\
After surgery & & & \\
$\quad$ 2 hours & $197.5 \pm 40.0$ & $212.1 \pm 44.0$ & 0.022 \\
Postoperative day 1 & & & \\
1st & $172.7 \pm 45.2$ & $173.4 \pm 51.4$ & 0.924 \\
2nd & $204.5 \pm 51.5$ & $209.1 \pm 54.9$ & 0.605 \\
3rd & $209.8 \pm 46.9$ & $196.1 \pm 47.9$ & 0.100 \\
4th & $191.9 \pm 46.4$ & $184.9 \pm 46.5$ & 0.379 \\
Postoperative day 2 & & & \\
1st & $162.0 \pm 35.1$ & $157.0 \pm 39.3$ & 0.425 \\
2nd & $203.0 \pm 56.8$ & $202.8 \pm 51.2$ & 0.979 \\
3rd & $192.1 \pm 46.8$ & $193.4 \pm 49.5$ & 0.873 \\
4th & $182.7 \pm 52.8$ & $180.8 \pm 52.1$ & 0.830 \\
\hline
\end{tabular}

Data are presented as the mean \pm standard deviation.

${ }^{a)}$ Group P, propofol; ${ }^{\text {b) }}$ Group S, sevoflurane.

Table 4. Postoperative outcomes

\begin{tabular}{lccc}
\hline & Group $\mathrm{P}^{\mathrm{a})}$ & Group S) & \\
& $(\mathrm{n}=89)$ & $(\mathrm{n}=87)$ & $p$-value \\
\hline Surgical site infection (n) & $0(0)$ & $2(2.3)$ & 0.243 \\
Hemodialysis (n) & $1(1.1)$ & $2(2.3)$ & 0.618 \\
Myocardial infarction (n) & $0(0)$ & $1(1.1)$ & 0.494 \\
Cerebral infarction (n) & $0(0)$ & $2(2.3)$ & 0.243 \\
ARDS (n) & $2(2.2)$ & $1(1.1)$ & 0.574 \\
Length of hospital stay (days) & $10(8-14)$ & $9(7-12)$ & 0.162 \\
In-hospital mortality (n) & $1(1.1)$ & $3(3.4)$ & 0.365 \\
30-day mortality (n) & $0(0)$ & $2(2.3)$ & 0.243 \\
\hline
\end{tabular}

Data are presented as the number (\%) or median (interquartile range).

ARDS, adult respiratory distress syndrome.

${ }^{a)}$ Group P, propofol; ${ }^{\text {b) }}$ Group S, sevoflurane.

short-term mortality compared with sevoflurane anesthesia in these patients.

Previous experimental studies have reported that sevoflurane and propofol induce insulin resistance, even in absence of surgical stress $[12,16,17]$. Under surgical stress, blood glucose levels are markedly increased with sevoflurane anesthesia but are relatively stable with propofol anesthesia [11]. The difference may be attributable to the different effects on ade- nosine triphosphate-sensitive potassium (KATP) channels in pancreatic $\beta$-cells - more specifically, glucose-stimulated insulin secretion is inhibited by sevoflurane [6,18], but not propofol $[10,12]$. These observations in experimental settings suggest that the choice of general anesthetic agent may affect glycemic management in the perioperative period, which is associated with postoperative morbidity and mortality. Kitamura et al. [14] demonstrated that propofol triggered lower blood glucose levels than sevoflurane during surgery in non-DM patients undergoing different types of surgery. However, becaus e insulin resistance in response to surgical stress is more pronounced in the postoperative period and may persist for several days following surgery [19], blood glucose level measurement confined to the intraoperative period may be inadequate to demonstrate the superiority of propofol over sevoflurane for glycemic control in surgical patients.

Lung surgery performed during OLV is accompanied by significant stress-induced endocrine response, as demonstrated in other type of major surgery, which may increase blood glucose levels during the perioperative period [20]. Regardless of general anesthetic, glucose levels were comparable during surgery but continually increased after surgery compared with preoperative values in surgical patients. Our results, however, are inconsistent with previous studies that have reported the maintenance of more stable blood glucose levels during propofol anesthesia than volatile anesthesia in surgical patients. Kitamura et al. [14] reported significant differences in blood glucose levels during surgery between sevoflurane and propofol anesthesia in non-DM surgical patients. However, the use of higher fentanyl doses during propofol anesthesia than sevoflurane anesthesia, the existence of various degrees of surgical stimulation, and the lack of anesthetic depth monitoring during surgery, may have obscured the lower glucose levels during propofol anesthesia compared with sevoflurane anesthesia in their retrospective study. Although several prospective studies have also reported lower blood glucose levels during propofol anesthesia versus volatile anesthesia, the clinical benefit of anesthetic choice on perioperative glycemic control remains controversial due to the absence of surgical stimulation [21], maintenance of euglycemia irrespective of anesthetic, or lack of glucose data from PODs ( $<72$ hours) [17,25-27], which is an important contributor to hyperglycemia-induced complications in surgical patients [24,25]. In the present study, sevoflurane and propofol anesthesia 
induced glycemic responses to major thoracic surgery maintained with comparable remifentanil dose and anesthetic depth. In addition, although glucose levels were significantly lower 2 hours postoperatively in patients with propofol anesthesia versus those with sevoflurane anesthesia, the incidence of perioperative hyperglycemia and clinical outcome were not different. The lack of clinical benefit from the relatively lower blood glucose levels in patients with propofol anesthesia in the immediate postoperative period may be explained by the multifactorial nature of perioperative hyperglycemia. A potential explanation may be that only severe hyperglycemia (glucose levels $>180 \mathrm{mg} / \mathrm{dL}[10.0 \mathrm{mmol} / \mathrm{L}]$ ) is a risk factor for mortality in DM patients with chronic exposure to increased glucose levels in contrast to non-DM patients [28]. The incidence of perioperative hyperglycemia was documented in more than two-thirds of our patients immediately after lung surgery with both sevoflurane and propofol anesthesia. Therefore, the acute effect of general anesthetics on blood glucose levels may contribute to severe hyperglycemia in DM patients with pre-existing high glucose levels due to major surgical stress.

Hyperglycemia has long been associated with adverse clinical outcomes, including death, caused by its deleterious effects on innate immune defenses. Hyperglycemia increases the risk for infection by impairing leukocyte function, including decreased phagocytosis of bacteria, reduced chemotaxis and generation of reactive oxygen species, and by compromised wound healing due to impaired collagen synthesis, reduced wound tensile strength, and reduced neovascularization [29]. Hyperglycemia also effects multi-organ dysfunction through endothelial dysfunction with decreasing vasodilation, increased vascular permeability, and impaired complement function in tissue injury. Acute perioperative hyperglycemia is associated with adverse clinical outcomes in non-cardiac surgery patients independent of DM [30]. However, no data are available on whether the effect of general anesthetics on blood glucose levels is associated with clinical outcome.

DM patients exhibit an overall higher rate of adverse events than non-DM patients after non-cardiac surgery [31]. However, DM patients with increased tolerance for higher blood glucose levels induce distinctly different implications and consequences of poor glycemic control compared with non-DM patients throughout the perioperative period $[3,27,28]$. While even a modest degree of hyperglycemia is a strong risk factor for superficial SSI, sepsis, and mortality in patients without a history of DM, only severe hyperglycemia is a risk factor for mortality and superficial SSI in patients with confirmed DM $[28,32]$. Hence, comparable incidence of severe hyperglycemia, despite different changes in blood glucose levels during the immediate postoperative period, may explain the comparable clinical outcomes in these patients.

There were several limitations to the present study, the first of which was its retrospective design. There may have been missing information on confounding factors affecting blood glucose levels during the perioperative period. Glycated hemoglobin ( $\mathrm{HbA1c}$ ) levels can identify DM patients with poor glycemic control over the previous several months and contribute to 30-day postoperative mortality and prolonged hospital stay $[31,33]$. Relative to HbA1c, random blood glucose levels or fasting blood glucose levels on the morning of surgery have limited ability to predict suboptimal control of blood glucose levels during the perioperative period. Although preoperative HbA1c level should be determined in all patients undergoing major surgery and in all elective surgical patients with DM [34], it was only available for a small proportion of patients in this study. Second, glucose monitoring during the perioperative period was not consistent. Blood glucose levels were determined using an on-site blood gas analyzer for intraoperative arterial samples, and by point-of-care capillary meters using a capillary sample postoperatively. Third, pain scores, which can affect glucose levels by modulating the endocrine stress response, were not included in this study because these data were not available in the medical records. Despite the equal distribution of two different analgesic techniques in our patients, postoperative pain control may contribute significantly to the postoperative glucose response.

In conclusion, both sevoflurane and propofol anesthesia were associated with a comparable incidence of hyperglycemia and clinical outcomes in patients with type 2 DM undergoing lung surgery. Prospective clinical trials are needed to examine the association between the effects of anesthetic agents on glucose metabolism and the clinical consequences in patients at risk for hyperglycemia during the perioperative period.

\section{CONFLICT OF INTEREST}

No potential conflict of interest relevant to this article was reported. 


\section{ORCID}

Hyuckgoo Kim, https://orcid.org/0000-0002-3879-6441

Jisoo Han, https://orcid.org/0000-0003-3611-0773

Sung Mee Jung, https://orcid.org/0000-0001-5602-9011

Sang-Jin Park, https://orcid.org/0000-0002-4838-2664

Nyeong Keon Kwon, https://orcid.org/0000-0003-0493-2203

\section{REFERENCES}

1. Desborough JP. The stress response to trauma and surgery. Br J Anaesth 2000;85:109-17.

2. Thorell A, Efendic S, Gutniak M, Häggmark T, Ljungqvist O. Development of postoperative insulin resistance is associated with the magnitude of operation. Eur J Surg 1993;159: 593-9.

3. Kotagal M, Symons RG, Hirsch IB, Umpierrez GE, Dellinger EP, Farrokhi ET, et al. Perioperative hyperglycemia and risk of adverse events among patients with and without diabetes. Ann Surg 2015;261:97-103.

4. Wu TY, Putaala J, Sharma G, Strbian D, Tatlisumak T, Davis SM, et al. Persistent hyperglycemia is associated with increased mortality after intracerebral hemorrhage. J Am Heart Assoc 2017;6. pii: e005760.

5. Akhtar S, Barash PG, Inzucchi SE. Scientific principles and clinical implications of perioperative glucose regulation and control. Anesth Analg 2010;110:478-97.

6. Zuurbier CJ, Keijzers PJ, Koeman A, Van Wezel HB, Hollmann MW. Anesthesia's effects on plasma glucose and insulin and cardiac hexokinase at similar hemodynamics and without major surgical stress in fed rats. Anesth Analg 2008;106:13542.

7. Ewart RB, Rusy BF, Bradford MW. Effects of enflurane on release of insulin by pancreatic islets in vitro. Anesth Analg 1981;60:878-84.

8. Tanaka T, Nabatame H, Tanifuji Y. Insulin secretion and glucose utilization are impaired under general anesthesia with sevoflurane as well as isoflurane in a concentration-independent manner. J Anesth 2005;19:277-81.

9. Wang L, Ko KW, Lucchinetti E, Zhang L, Troxler H, Hersberger $\mathrm{M}$, et al. Metabolic profiling of hearts exposed to sevoflurane and propofol reveals distinct regulation of fatty acid and glucose oxidation: CD36 and pyruvate dehydrogenase as key regulators in anesthetic-induced fuel shift. Anesthesiology 2010;113:541-51.

10. Tanaka K, Kawano T, Tsutsumi YM, Kinoshita M, Kakuta $\mathrm{N}$, Hirose K, et al. Differential effects of propofol and isoflurane on glucose utilization and insulin secretion. Life Sci 2011; 88:96-103.

11. Kitamura T, Ogawa M, Kawamura G, Sato K, Yamada Y. The effects of sevoflurane and propofol on glucose metabolism under aerobic conditions in fed rats. Anesth Analg 2009;109: 1479-85.
12. Li X, Kitamura T, Kawamura G, Mori Y, Sato K, Araki Y, et al. Comparison of mechanisms underlying changes in glucose utilization in fasted rats anesthetized with propofol or sevoflurane: Hyperinsulinemia is exaggerated by propofol with concomitant insulin resistance induced by an acute lipid load. Biosci Trends 2014;8:155-62.

13. Cok OY, Ozkose Z, Pasaoglu H, Yardim S. Glucose response during craniotomy: propofol-remifentanil versus isofluraneremifentanil. Minerva Anestesiol 2011;77:1141-8.

14. Kitamura T, Kawamura G, Ogawa M, Yamada Y. Comparison of the changes in blood glucose levels during anesthetic management using sevoflurane and propofol. Masui 2009;58:81-4.

15. Bilotta F, Giovannini F, Caramia R, Rosa G. Glycemia management in neurocritical care patients: a review. J Neurosurg Anesthesiol 2009;21:2-9.

16. Yasuda Y, Fukushima Y, Kaneki M, Martyn JA. Anesthesia with propofol induces insulin resistance systemically in skeletal and cardiac muscles and liver of rats. Biochem Biophys Res Commun 2013;431:81-5.

17. Kim SP, Broussard JL, Kolka CM. Isoflurane and sevoflurane induce severe hepatic insulin resistance in a canine model. PLoS One 2016;11:e0163275.

18. Saho S, Kadota Y, Sameshima T, Miyao J, Tsurumaru T, Yoshimura N. The effects of sevoflurane anesthesia on insulin secretion and glucose metabolism in pigs. Anesth Analg 1997; 84:1359-65.

19. Thorell A, Efendic S, Gutniak M, Häggmark T, Ljungqvist O. Insulin resistance after abdominal surgery. Br J Surg 1994; 81:59-63.

20. Tønnesen E, Höhndorf K, Lerbjerg G, Christensen NJ, Hüttel MS, Andersen K. Immunological and hormonal responses to lung surgery during one-lung ventilation. Eur J Anaesthesiol 1993;10:189-95.

21. Jeong JS, Oh SW, Koo GH. Comparison of effects of propofol and enflurane on blood glucose level. Korean J Anesthesiol 1998;34:323-8. Korean.

22. Kumar M, Tripathi M, Malviya D, Malviya PS, Kumar V, Tyagi A. Influence of two anesthetic techniques on blood sugar level in head injury patients: a comparative study. Anesth Essays Res 2016;10:207-11.

23. Behdad S, Mortazavizadeh A, Ayatollahi V, Khadiv Z, Khalilzadeh S. The effects of propofol and isoflurane on blood glucose during abdominal hysterectomy in diabetic patients. Diabetes Metab J 2014;38:311-6.

24. McConnell YJ, Johnson PM, Porter GA. Surgical site infections following colorectal surgery in patients with diabetes: association with postoperative hyperglycemia. J Gastrointest Surg 2009;13:508-15.

25. Mahid SS, Polk HC Jr, Lewis JN, Turina M. Opportunities for improved performance in surgical specialty practice. Ann Surg 2008;247:380-8.

26. Clarke RS. The hyperglycaemic response to different types of surgery and anaesthesia. Br J Anaesth 1970;42:45-53.

27. Frisch A, Chandra P, Smiley D, Peng L, Rizzo M, Gatcliffe C, et al. Prevalence and clinical outcome of hyperglycemia in the perioperative period in noncardiac surgery. Diabetes 
Care 2010;33:1783-8.

28. Krinsley JS. Glycemic control, diabetic status, and mortality in a heterogeneous population of critically ill patients before and during the era of intensive glycemic management: six and one-half years experience at a university-affiliated community hospital. Semin Thorac Cardiovasc Surg 2006;18:31725 .

29. Turina M, Fry DE, Polk HC Jr. Acute hyperglycemia and the innate immune system: clinical, cellular, and molecular aspects. Crit Care Med 2005;33:1624-33.

30. Kwon S, Thompson R, Dellinger P, Yanez D, Farrohki E, Flum D. Importance of perioperative glycemic control in general surgery: a report from the Surgical Care and Outcomes Assessment Program. Ann Surg 2013;257:8-14.

31. Yeh CC, Liao CC, Chang YC, Jeng LB, Yang HR, Shih CC, et al. Adverse outcomes after noncardiac surgery in patients with diabetes: a nationwide population-based retrospective cohort study. Diabetes Care 2013;36:3216-21.

32. Mohan S, Kaoutzanis C, Welch KB, Vandewarker JF, Winter S, Krapohl G, et al. Postoperative hyperglycemia and adverse outcomes in patients undergoing colorectal surgery: results from the Michigan surgical quality collaborative database. Int J Colorectal Dis 2015;30:1515-23.

33. Underwood P, Askari R, Hurwitz S, Chamarthi B, Garg R. Preoperative $\mathrm{A} 1 \mathrm{C}$ and clinical outcomes in patients with diabetes undergoing major noncardiac surgical procedures. Diabetes Care 2014;37:611-6.

34. Aldam P, Levy N, Hall GM. Perioperative management of diabetic patients: new controversies. Br J Anaesth 2014;113: 906-9. 